Journal of Innovative Optical Health Sciences

Vol. 11, No. 5 (2018) 1850022 (10 pages)

(C) The Author(s)

DOI: $10.1142 / \mathrm{S} 1793545818500220$

\title{
A photoacoustic imaging system with variable gain at different depths
}

\author{
Tian Guan*,†, Yao Li*,†, Muqun Yang ${ }^{\star}$, Yong Jiang ${ }^{\S}$ \\ and Yonghong $\mathrm{He}^{*}$ \\ *Shenzhen Key Laboratory for Minimal \\ Invasive Medical Technologies \\ Graduate School at Shenzhen \\ Tsinghua University, Shenzhen 518055, P. R. China \\ $\dagger$ Department of Biomedical Engineering \\ Tsinghua University, Beijing 100084, P. R. China \\ $\star$ Center of Precision Medicine and Healthcare \\ Tsinghua-Berkeley Shenzhen Institute, Shenzhen 518055, P. R. China \\ ${ }^{\S}$ Shenzhen Wisonic Medical Technology Co., Ltd. \\ Taoyuan Street, Nanshan District, Shenzhen 518055, P. R. China \\ ॠguantian@sz.tsinghua.edu.cn
}

Received 28 February 2018

Accepted 26 April 2018

Published 2 August 2018

\begin{abstract}
We established a photoacoustic imaging (PAI) system that can provide variable gain at different depths. The PAI system consists of a pulsed laser with an optical parametric oscillator working at a $728 \mathrm{~nm}$ wavelength and an imaging-acquisition-and-processing unit with an ultrasound transducer. A voltage-controlled attenuator was used to realize variable gain at different depths when acquiring PAI signals. The proof-of-concept imaging results for variable gain at different depths were achieved using specific phantoms. Both resolution and optical contrast obtained through the results of variable gain for a targeted depth range are better than those of constant gain for all depths. To further testify the function, we imaged the sagittal section of the body of in vivo nude mice. In addition, we imaged an absorption sample embedded in a chicken breast tissue, reaching a maximum imaging depth of $\sim 4.6 \mathrm{~cm}$. The results obtained using the proposed method showed better resolution and contrast than when using $50 \mathrm{~dB}$ gain for all depths. The depth range resolution was $\sim 1 \mathrm{~mm}$, and the maximum imaging depth of our system reached $\sim 4.6 \mathrm{~cm}$. Furthermore, blood vessels can be revealed and targeted depth range can be selected in nude mice imaging.
\end{abstract}

Keywords: Photoacoustic imaging; variable gain; imaging depth.

TCorresponding author.

This is an Open Access article published by World Scientific Publishing Company. It is distributed under the terms of the Creative Commons Attribution 4.0 (CC-BY) License. Further distribution of this work is permitted, provided the original work is properly cited. 


\section{Introduction}

Photoacoustic imaging (PAI) is a new technique that is gaining considerable attention in biomedical engineering. It is a hybrid imaging technique that combines optical imaging with ultrasound imaging (USI). ${ }^{1,2}$ The principles of PAI are based on the photoacoustic effect discovered by Bell in 1880. ${ }^{3}$ The imaging process includes the following: (1) pulsed laser with a certain wavelength stimulating the optical absorber as the object, (2) thermal expansion of the object after absorption, (3) ultrasound (US) waves regarded as photoacoustic (PA) signals spreading in all directions, (4) PA signal collection, and (5) image reconstruction. Compared with USI, PAI can provide better image resolution. ${ }^{1}$ In addition, the scattering of a PA signal is much inferior to light when it propagates through biotic tissues. Therefore, PAI is supposed to offer better penetration depth, normally of several centimeters. ${ }^{4,5}$

An outstanding feature of PAI is that PA images can be reconstructed based on the endogenous optical absorption characteristic of different tissues without the specific exogenous contrast agents. ${ }^{6}$ In 2003, Wang et al. released the structure and function images of rat brain via PA tomography. ${ }^{7}$ Many imaging systems based on PAI have been set up for specific objects, such as small animals, ${ }^{8}$ human breasts,,${ }^{9,10}$ and sentinel lymph nodes. ${ }^{11,12}$

In conventional USI systems, the attenuation caused by the strong scattering of tissues is supposed to result in inhomogeneous image resolution and low signal-to-noise ratio (SNR). ${ }^{13}$ Sophisticated techniques to compensate for signal attenuation in USI systems have already been developed. To improve the SNR of a US image, signal modulation and matched filtering were used. ${ }^{14}$ Jung et al. applied a low-noise amplifier using attenuationadaptive noise control to a USI to reduce the useless noise floor. ${ }^{15}$ As the process of signal acquisition of PAI is similar to that of USI, techniques in USI can be utilized in PAI for compensating for signal attenuation. In USI, assuming imaging sample is uniform, the attenuation occurs with the process of US transmission and reflection. Thus, USI system provides compensation for US signal attenuation. In PAI, on one hand, PAI system does not need to transmit US, and PA signal spreads from absorbers in imaging sample. So, the process of compensating for PA signal attenuation is the same as US reflection in USI. On the other hand, PAI has to compensate for the attenuation of the light penetration in sample. Its parameters are different. Two parts work together as gain compensation in this PAI system.

In this paper, we established a PAI system with variable gain $(0-50 \mathrm{~dB})$ in signal acquisition for different depths. This function can provide variable gain to original analogue signals during signal acquisition which can maximize the restoration of all signals from real environment without distortions. We implemented variable gain for different depths by using a voltage-controlled attenuator to compensate PA signal attenuation from different depths of the imaging sample. By imaging specific phantoms, we tested the implementation of the technique of variable gain at different depths. The proposed technique brings better resolution and optical contrast to PA images. In addition, to quantify the imaging depth of the PAI system, we imaged chicken breast tissue as the biotic sample and optical absorber. The results reveal the possible application of our PAI system to the in vivo animal imaging and even human tissue imaging in further studies.

\section{Methods and Materials}

\subsection{System setup}

Figure 1 shows the schematic graph of the whole PAI system. The system consists of a pulsed laser, an optical parametric oscillator (OPO), an imaging acquisition and processing unit, a commercial US transducer, and a PC. The system is able to achieve the functions of pulsed laser stimulation, PA signal acquisition, and PA image reconstruction.

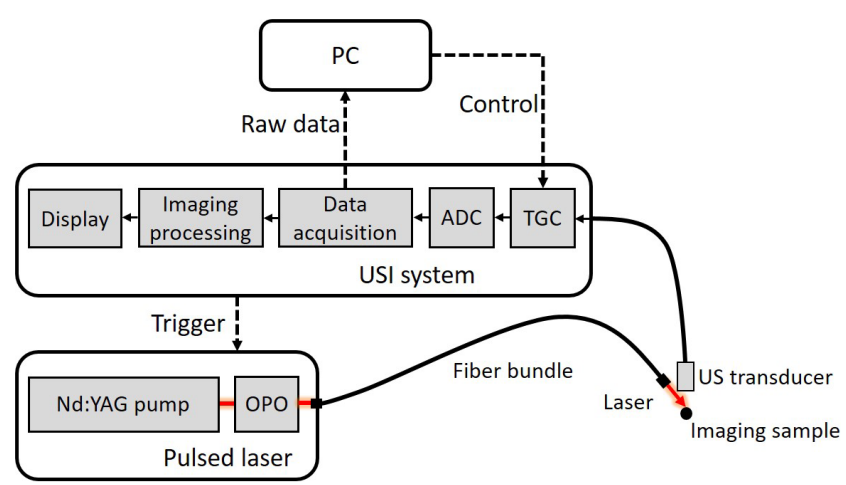

Fig. 1. Schematic graph of the PAI system. TGC: time-gaincompensation. 
The pulsed laser (Dawa-300, Beamtech Optronics Co., Ltd., China) we utilized can provide a Qswitched Nd:YAG pump laser. The intensity and internal trigger repetition frequency of the laser were controlled by using the handheld controller. The original wavelength of the output light from the laser pumping source was $532 \mathrm{~nm}$. The prepositioned OPO (BB-OPO-725, Dalian Huayang Science \& Technology Co., Ltd., China) and its assorted software modified the laser wavelength to $728 \mathrm{~nm}$. The process of PAI relies on the thermal expansion of optical absorbers under the excitation of the laser. So, high scattering of the laser in tissues is beneficial to imaging results. Comparing with higher wavelength, laser with wavelength of $728 \mathrm{~nm}$ can provide less absorbance but more scattering for tissues, which is appropriate for this system.

We applied a portable clinical USI system (Clover, Shenzhen Wisonic Medical Technology Co., Ltd., China) to our PAI system. The USI system can present real-time US images on the screen. Through the integrated control panel of the system, necessary information can be displayed and important operations can be completed. In addition, a commercial linear array US transducer (L15-4, Shenzhen Wisonic Medical Technology Co., Ltd., China), comprising 64 channels, was used for PA signal acquisition. The console of the USI system can turn off ultrasonic emission under the condition of obtaining PA signals.

To synchronize the laser and USI systems, we set the laser system into the external-trigger mode. The console of the USI system closes ultrasonic emission and simultaneously sends a $+5 \mathrm{~V}$ trigger signal to the laser system with a $10 \mathrm{~Hz}$ frequency. Thus, the repetition of the pulsed laser was $10 \mathrm{~Hz}$ in our PAI system.

The USI system was equipped with client software installed on a PC (230X, Lenovo Group Ltd., China). By using a wireless network, the software was used to optimize the parameters of the USI system and control PA data acquisition. During signal acquisition, the original analog signal was amplified through amplifiers and sampled using a 14-bit analog-to-digital convertor (ADC) module with a sampling rate of $40 \mathrm{MHz}$.

Then, the 64-channel linear array US transducer with a central frequency of $8 \mathrm{MHz}$ was used to acquire the raw data from the PA signal. After processing the raw data by using a band-pass filter with a central frequency of $4 \mathrm{MHz}, \mathrm{PA}$ images were reconstructed based on the delay-and-sum (DAS) algorithm. The program was developed through Matlab on the PC. The process of image acquisition and processing unit is demonstrated in Fig. 2(a).

\subsection{Method of variable gain for different depths}

The implementation of variable gain for different depths is based on the usage of the voltage-controlled attenuator, which possesses linear attenuation characteristics, because of which the average gain loss is constant for each equal increment of the control voltage ${ }^{16}$ The attenuator is a variable voltage divider comprised of a series of input resistors $\left(R_{s}\right)$ and parallel-connected field effect transistors (FETs). FETs are controlled using a sequentially activated clipper amplifier $\left(A_{1}-A_{n}\right)$. Figure 2(c) presents the design of the attenuator.

With the increase of the input control voltage, the FET changes from closing state to fully open state. When each FET enters the open state and the control voltage continues to increase, the next amplifier and FET start functioning, showing the linear attenuation characteristic of the next part. Therefore, the low control voltage causes the FET to turn off, and the attenuator exhibits minimum signal attenuation. Similarly, high control voltage causes the FET to switch on, and the attenuator shows maximum signal attenuation.

The implementation process of providing variable gain for different depths is shown in Fig. 2(b). During signal acquisition, we set different digital input data as different gains. As PA signals from different depths in the imaging sample have different time delays, we vary the trigger time of control voltage acting on the attenuator. So, the control voltage is bound to different depth. By using the 8-bit digital-to-analog converter (DAC) module with a sampling rate of $1 \mathrm{MHz}$, the signal attenuation of the attenuator was regulated. Thus, the gain at different depths of the PA signal acquisition is under control.

\subsection{Experimental process}

We developed a $U$ mold through three-dimensional (3D) printing by using photopolymer as the source material to fix black hairs. The left and right arms of the mold were evenly spaced with $0.5 \mathrm{~cm}$ holes, and the diameter of each hole was $0.5 \mathrm{~mm}$. Next, the 


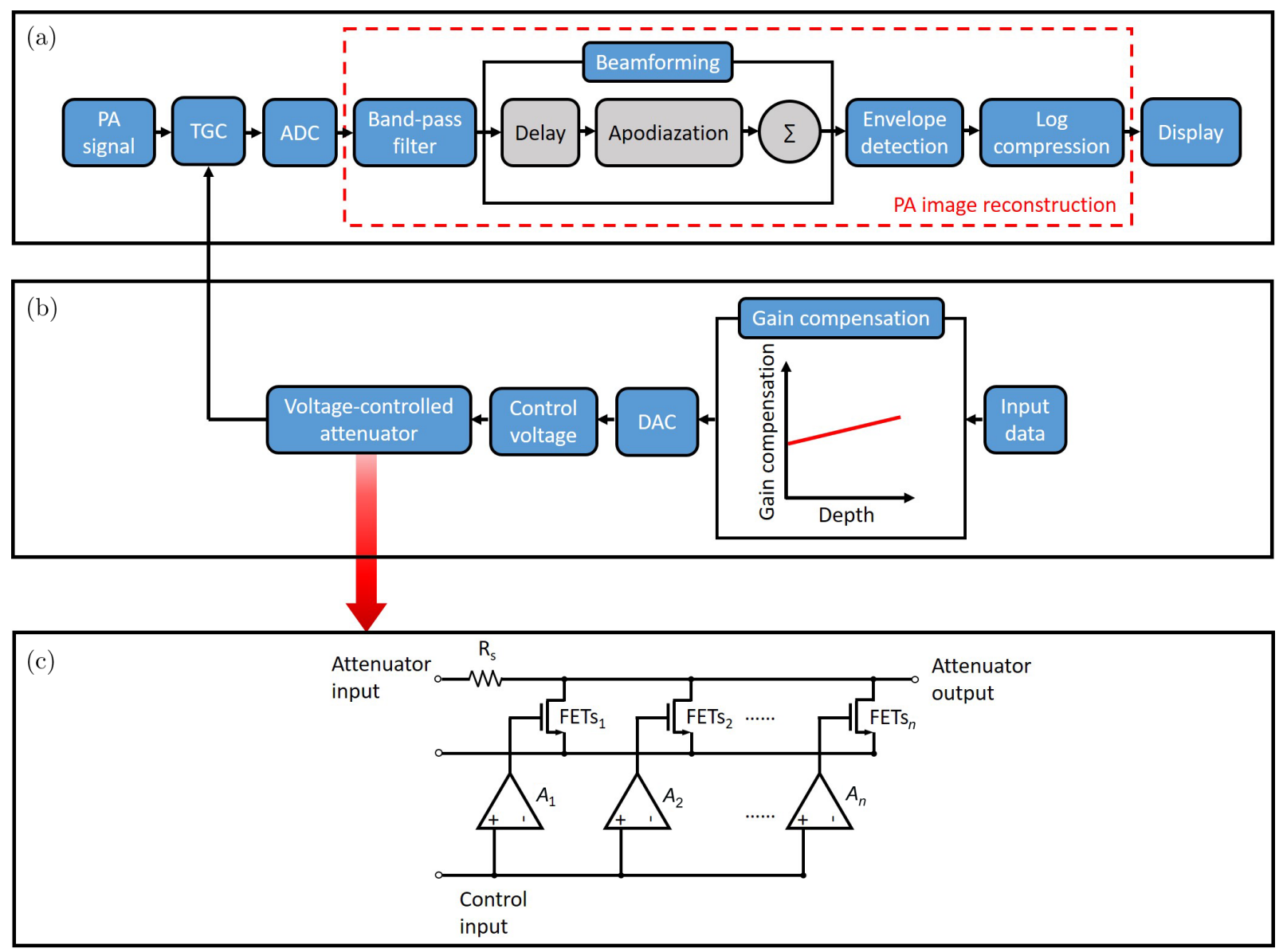

Fig. 2. Schematic of the imaging acquisition and processing unit: (a) signal acquisition and image reconstruction, (b) variable gain providing, and (c) the design of the attenuator. A: clipper amplifiers.

hairs were aligned through two adjusted holes, straightened, and fixed. We put the mold with hairs into a $500-\mathrm{mL}$ beaker. Then, we heated up $1 \%$ agarose emulsion to obtain a transparent solution and slowly poured it into the beaker containing the mold. By controlling the amount of added agarose solution, we guaranteed that there were two hairs $0.5 \mathrm{~cm}$ apart in a depth range of $1 \mathrm{~cm}$. As shown in Fig. 3(a), after the agarose solution was cooled down, a scattering medium was formed around the fixed hairs. We measured the intensity of incident light and the intensity of the scattering light through the phantom to address the scattering coefficient of the scattering medium. The scattering coefficient is $\sim 0.4$ and the laser was scattered evenly.

To evaluate the maximum imaging depth of the PAI system, we imaged a 1-mL centrifugal tube filled with pure black ink. We developed a specific biotic sample with chicken breast tissue because the optical property of chicken breast tissue is comparable to human tissue. ${ }^{17,18}$ For the scattering of the tissue is homogeneous, the laser can be scattered uniformly in the phantom. To avoid the interference of ultrasonic reflection, we placed $\sim 5-\mathrm{cm}$ thick chicken breast tissue at the bottom of the 500$\mathrm{mL}$ beaker [Fig. 5(a)]. Then, we placed the centrifugal tube on the tissue pile. During the experiment, we stacked the chicken breast tissue layer-by-layer upon the tube so that the tube could be imaged at different depths.

To further testify the implementation of variable at different depths, we applied the PAI system to in vivo animal imaging. All procedures were conducted in accordance with the guidelines of the Administrative Committee on Animal Research at the Graduate School at Shenzhen, Tsinghua University. We used healthy BALB/c-nu/nu female nude mice which were five-week-old, weighing 14-16 g. During experiments, the mouse was anaesthetized by injection of $10 \%$ chloral hydrate solution. To keep the mouse's body 


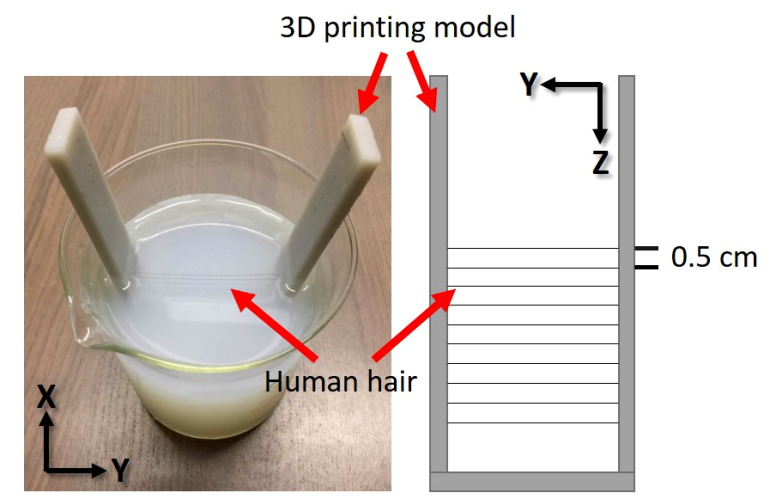

(a)
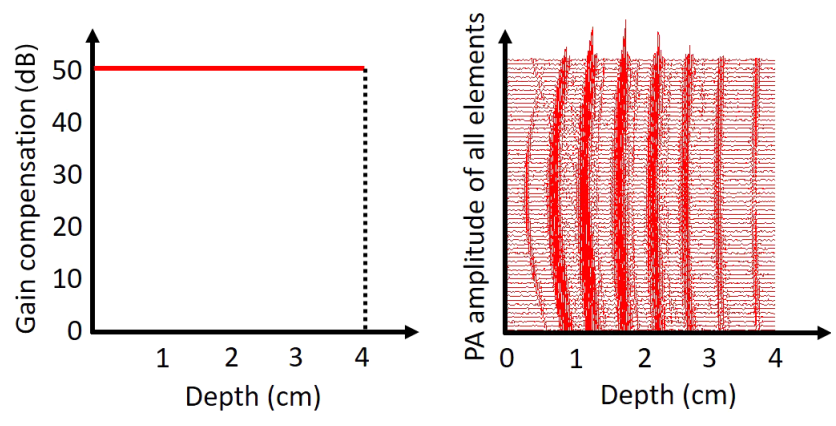

(b)
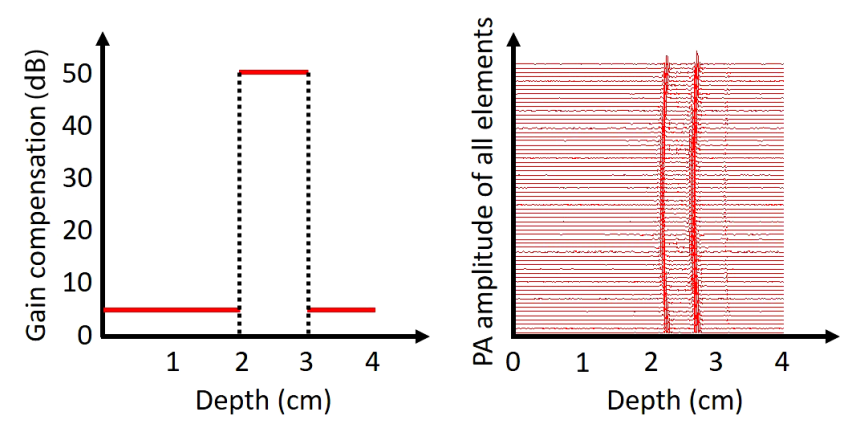

(c)
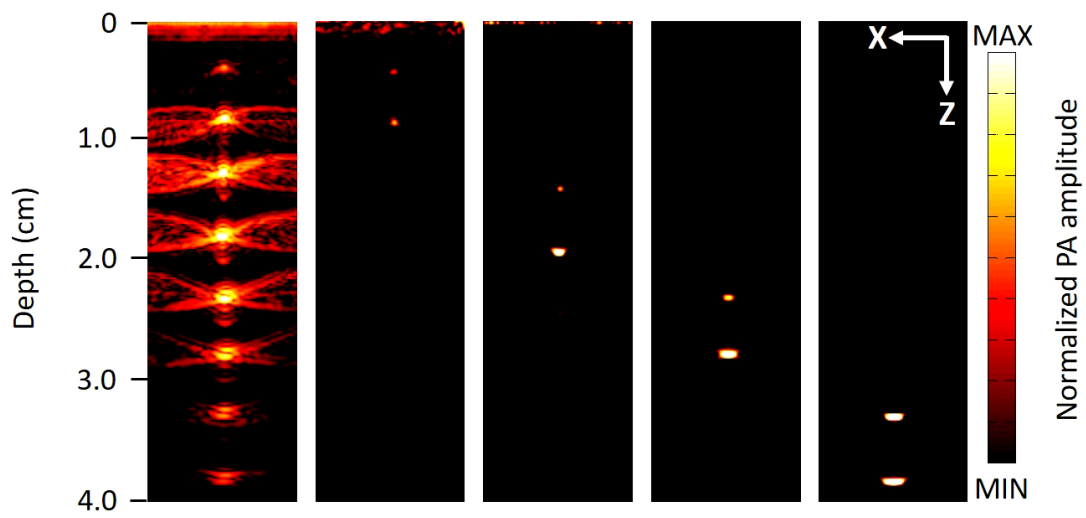

(d)

Fig. 3. Experiments for variable gain at different depths. (a) Actual photo of the phantom and schematic graph of the $U$ mold; (b) gain compensation of $50 \mathrm{~dB}$ for all depths (left) and original PA signals (right); (c) gain compensation of $50 \mathrm{~dB}$ for $2-3 \mathrm{~cm}$ depth range (left) and original PA signals (right); (d) PA images, from left to right: $50 \mathrm{~dB}$ at all depths; $50 \mathrm{~dB}$ at the $0-1 \mathrm{~cm}$ depth range, $5 \mathrm{~dB}$ at others; $50 \mathrm{~dB}$ at the $1-2 \mathrm{~cm}$ depth range, $5 \mathrm{~dB}$ at others; $50 \mathrm{~dB}$ at the $2-3 \mathrm{~cm}$ depth range, $5 \mathrm{~dB}$ at others; and $50 \mathrm{~dB}$ at the $3-4 \mathrm{~cm}$ depth range, $5 \mathrm{~dB}$ at others.

temperature, it was placed on a heating pad, lying on the back. We used a gel pad and US coupling gel between imaging target and the US transducer to match acoustic impedance. The imaging target is shown in Fig. 6(a). After all imaging process, the mouse was sacrificed with an overdose injection of the anesthetic.
After turning on the laser system, we adjusted OPO to control the wavelength of the output light at $728 \mathrm{~nm}$. We utilized an optical fiber to guide the laser to the top of the imaging sample. Under the triggering of the USI system, the repetition frequency of the pulsed laser was $10 \mathrm{~Hz}$. By controlling the voltage of the laser system, the energy of the 


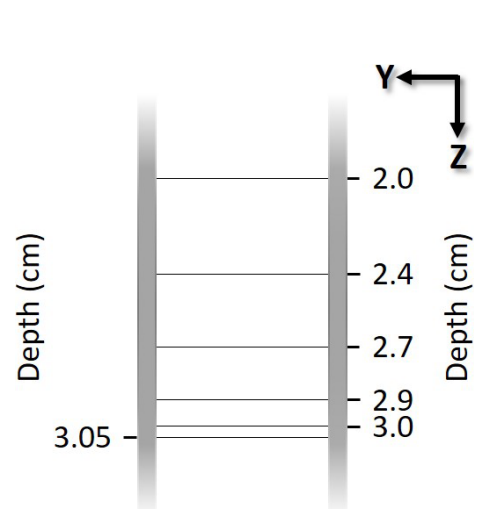

(a)
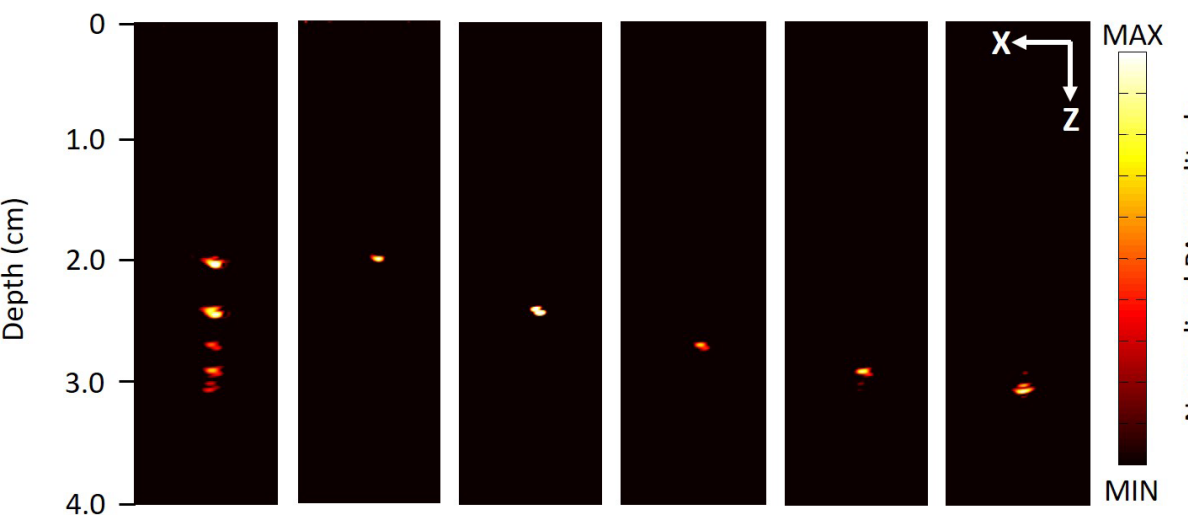

(b)

Fig. 4. Experiments for depth range resolution. (a) Schematic graph of the phantom and (b) PA images, from left to right: $50 \mathrm{~dB}$ at all depths; $50 \mathrm{~dB}$ at $2 \mathrm{~cm}$ depth, $5 \mathrm{~dB}$ at others; $50 \mathrm{~dB}$ at $2.4 \mathrm{~cm}$ depth, $5 \mathrm{~dB}$ at others; $50 \mathrm{~dB}$ at $2.7 \mathrm{~cm}$ depth, $5 \mathrm{~dB}$ at others; $50 \mathrm{~dB}$ at $2.9 \mathrm{~cm}$ depth, $5 \mathrm{~dB}$ at others; $50 \mathrm{~dB}$ at $3 \mathrm{~cm}$ depth, $5 \mathrm{~dB}$ at others.

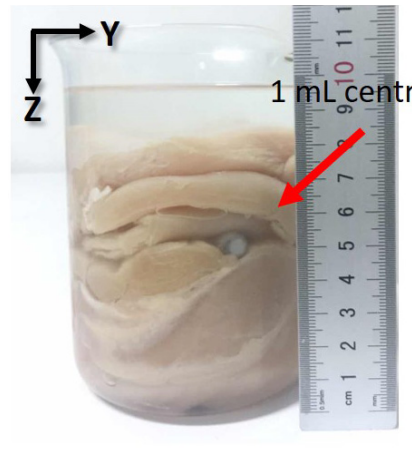

Side view

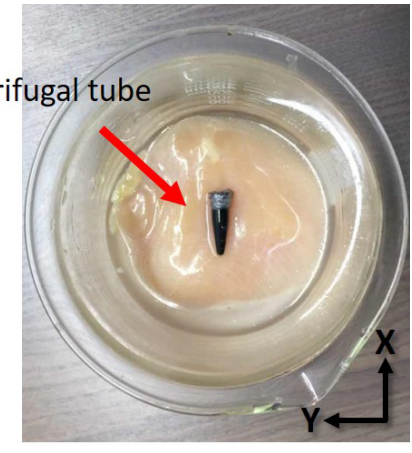

Top view

(a)
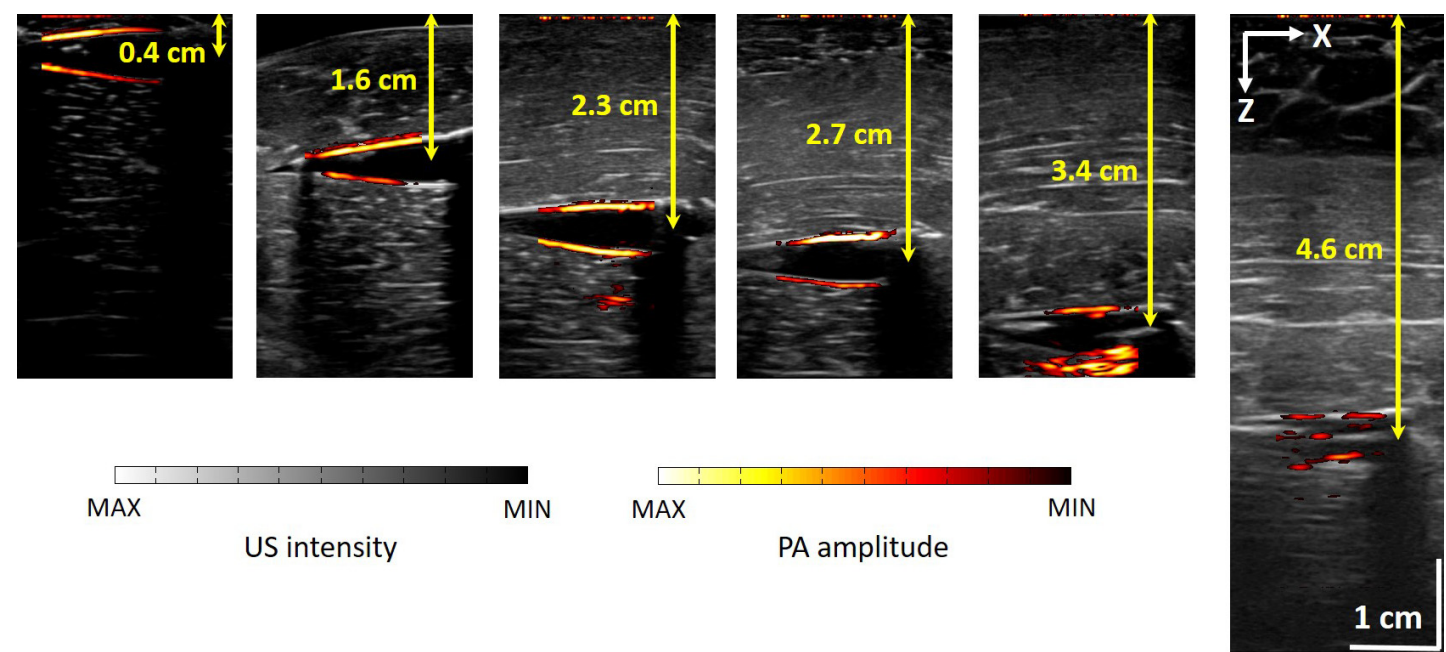

(b)

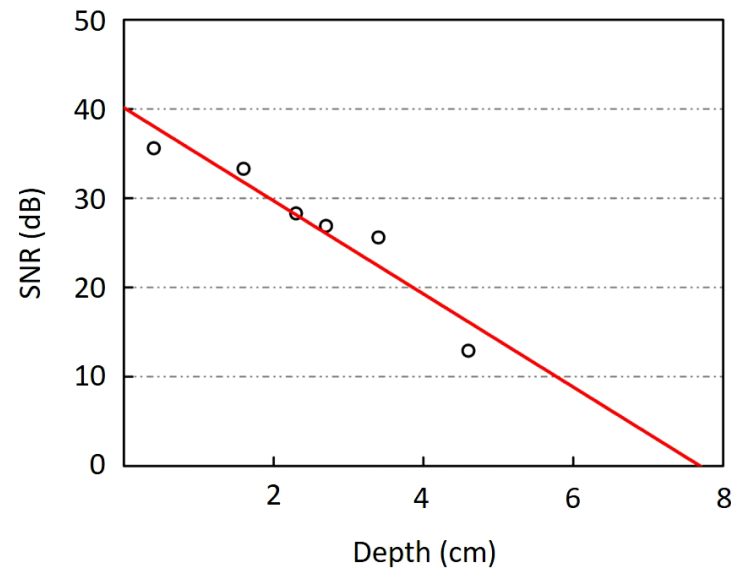

(c)
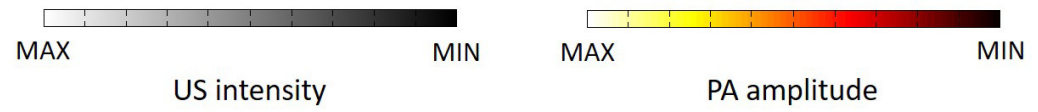

$\mathrm{cm}$

Fig. 5. Experiments for maximum imaging depth. (a) The actual photo of the phantom with chicken breast tissue. (b) The overlaid PA and US images for the centrifugal tube at different depths. (c) SNR of PA images for the centrifugal tube at different depths. 


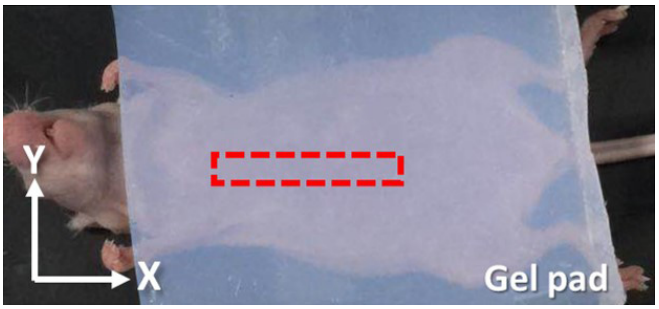

(a)

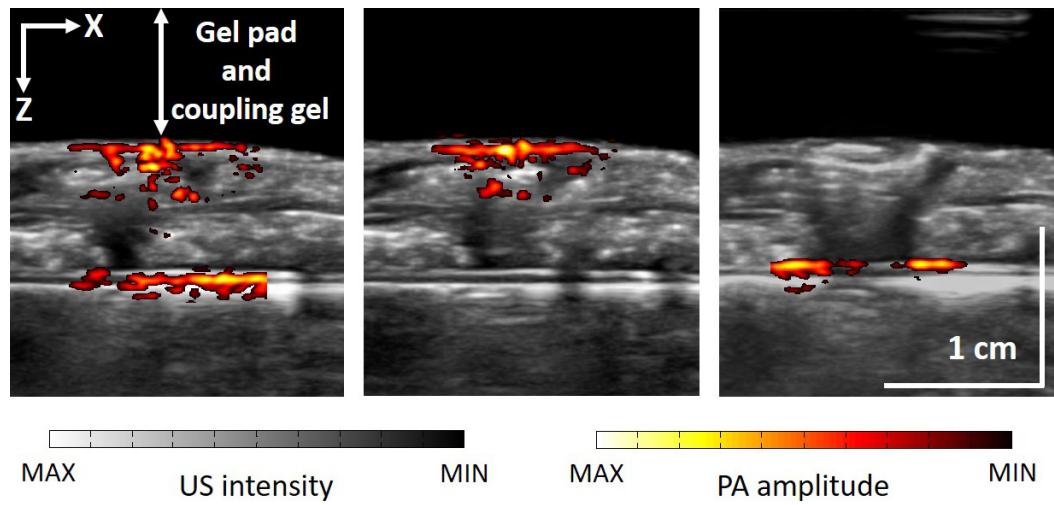

(b)

Fig. 6. Experiments for in vivo nude mice imaging. (a) Actual photo of the mouse and imaging region (the red dashed rectangle). (b) PA images, from left to right: $50 \mathrm{~dB}$ at all depths; $50 \mathrm{~dB}$ at the $0.6-1.2 \mathrm{~cm}$ depth range, $5 \mathrm{~dB}$ at others; $50 \mathrm{~dB}$ at the $1.5-2 \mathrm{~cm}$ depth range, $5 \mathrm{~dB}$ at others.

output light was approximately $15 \mathrm{~mJ} / \mathrm{cm}^{2}$, which is below the ANSI safety limitation at a wavelength of $728 \mathrm{~nm} .^{19}$

By using the 64-channel US transducer, raw data of the PA signal were obtained by using the imaging acquisition and processing unit. Then, the PA image was reconstructed based on the raw data by using the DAS algorithm.

\section{Results and Discussions}

\subsection{Variable gain at different depths}

After connecting the $\mathrm{PC}$ with the USI system via a wireless network, we regulated a $50 \mathrm{~dB}$ gain for all depths by using the software. The gain with depth and the original PA signals from $0-4 \mathrm{~cm}$ depth range are depicted in Fig. 3(b). As shown in the left-most image of Fig. 3(d), the PA signal amplitude of the cross-section of hair is well displayed after image reconstruction in pseudo color. As there is an angle of $\sim 30^{\circ}$ between the optical fiber and sample, the light intensity in the depth range of $0.5-3 \mathrm{~cm}$ is relatively high. Thus, the amplitude of the PA signal is correspondingly higher in this depth range.

Furthermore, we set different gains for different depths: $50 \mathrm{~dB}$ at the $0-1 \mathrm{~cm}$ depth range, $5 \mathrm{~dB}$ at others; $50 \mathrm{~dB}$ at the $1-2 \mathrm{~cm}$ depth range, $5 \mathrm{~dB}$ at others; $50 \mathrm{~dB}$ at the $2-3 \mathrm{~cm}$ depth range, $5 \mathrm{~dB}$ at others; $50 \mathrm{~dB}$ at the $3-4 \mathrm{~cm}$ depth range, $5 \mathrm{~dB}$ at others. By adjusting the gain, we can effectively display the cross-section of hairs only in the 1-cm depth range. From Fig. 3(c), the left picture describes the gain providing and the right one illustrates the original PA signals after signal acquisition. For the gain to be $50 \mathrm{~dB}$ only at that depth range, only hairs in $2-3 \mathrm{~cm}$ depth range can send effective signal, which well corresponds to the fourth image from the left in Fig. 3(d). No matter which targeted depth range was involved, the results showed much better axial and lateral resolutions, as well as optical contrast, compared with the results obtained using $50 \mathrm{~dB}$ gain for all depths. As the hair embeds deeper, the axial and lateral resolutions of the hair cross-section in the PA images increase, which is in accordance with the US images in the conventional USI.

As the distance between two adjacent hairs is $0.5 \mathrm{~cm}$, the minimum depth range for providing variable gain is less than $0.5 \mathrm{~cm}$. To further investigate the depth range resolution for providing variable gain, we improved the imaging sample based on the previously mentioned phantom. At 2 $3.05 \mathrm{~cm}$ depth range, we placed and fixed six hairs at a depth of $2,2.4,2.7,2.9,3$, and $3.05 \mathrm{~cm}$ on a $3 \mathrm{D}$ printing mold. Therefore, the spacing of hairs from top to bottom was $0.4,0.3,0.2,0.1$, and $0.5 \mathrm{~mm}$. Figure 4(a) shows the schematic graph of the sample, which was imaged using our PAI system. During signal acquisition, we set the gain of $50 \mathrm{~dB}$ for the aforementioned positions separately and gain of $5 \mathrm{~dB}$ for other depths.

The results are demonstrated in Fig. 4(b), where two hair sections in the PAI can be clearly seen to separate at a spacing of $0.4,0.3$, and $0.2 \mathrm{~mm}$. However, when the hair at a $2.9 \mathrm{~cm}$ depth was imaged with a gain of $50 \mathrm{~dB}$ ( $5 \mathrm{~dB}$ for others), the hair 
at $3 \mathrm{~cm}$ depth is slightly visible. Similarly, when the hair at $3 \mathrm{~cm}$ depth was imaged with a gain of $50 \mathrm{~dB}$ ( $5 \mathrm{~dB}$ for others), the hair at $2.9 \mathrm{~cm}$ depth is observed in the PA image. Therefore, the depth range resolution for providing variable gain can be regarded as $1 \mathrm{~mm}$ for our PAI system.

Moreover, the variable gain control for a smaller depth range allows for more targeted raw data to optimize the process of image reconstruction. Similarly, in Fig. 4(b), the image of the hair section with $50 \mathrm{~dB}$ gain only at $2,2.4,2.7,2.9$, and $3 \mathrm{~cm}$ shows better resolution and optical contrast.

\subsection{Maximum imaging depth}

To determine the maximum imaging depth of our PAI system, we imaged the centrifugal tube at the depths of $0.4,1.6,2.3,2.7,3.4$, and $4.6 \mathrm{~cm}$ by adding chicken tissue to cover the tube in the $500 \mathrm{~mL}$ beaker [Fig. 5(a)]. The gain was set at $50 \mathrm{~dB}$ for all depths. The overlaid US and PA images are depicted in Fig. 5(b). The US intensity is shown in a grayscale map, while the PA amplitude is presented in pseudo color.

Furthermore, SNR of the PA image was evaluated using the following formula: $\mathrm{SNR}=20 \mathrm{lg}$ $\left(V_{s} / V_{n}\right)$, where $V_{s}$ is the average value of the normalized PA signal amplitudes, while $V_{n}$ represents the standard deviation of the background signal. To obtain an accurate result, the average SNR of 10 PA images was evaluated at different depths, as shown in Fig. 5(c). The average SNR for six depths was obtained as $27.1 \mathrm{~dB}$.

With the increase of depth, the SNR decayed at $\sim 5.2 \mathrm{~dB} / \mathrm{cm}$. At the depth of $4.6 \mathrm{~cm}$, the PA signal can barely be distinguished from the background noise because of the penetration limit of the laser and the scattering of the environment. Therefore, the depth of $4.6 \mathrm{~cm}$ is considered to be the maximum imaging depth of the system with the SNR of $12.9 \mathrm{~dB}$. This result is consistent with those of other research $^{1,20,21}$ at the penetration depth of $4-5 \mathrm{~cm}$. The laser intensity is far beyond the safety limits at $15 \mathrm{~mJ} / \mathrm{cm}^{2}$ and the fiber bundle offers an energy loss during transmission. During the experiment, we used pure black ink instead of a specific dye, such as methylene blue, ${ }^{20}$ phosphorus phthalocyanine (P-Pc),${ }^{21}$ and indocyanine Green. ${ }^{22}$ In future experiments, the penetration and SNR are expected to be improved by using appropriate dye.

\subsection{In vivo animal imaging}

In this section, the PAI system were applied to living tissue imaging to assess its performance. And the implementation of the function of variable gain at different depths were further verified. We utilized the system on imaging of nude mice. The results are shown in Fig. 6(b). US images are in grayscale while PA images are in pseudo color.

From the left image of Fig. 6(b), the gains of all depths were set as $50 \mathrm{~dB}$. The blood vessels from upper and lower superficial skin are visible. Furthermore, hemoglobin possesses relatively high absorbance under the stimulation of the laser with the wavelength of $728 \mathrm{~nm}$. Therefore, the organ with blood is revealed beneath the skin of the mouse.

To further testify the function of variable gain at different depths, we set the gain of $50 \mathrm{~dB}$ at depth of $0.6-1.2 \mathrm{~cm}$, only the blood vessels from upper skin and parts of the tissue with blood are visible, shown as the image in the middle of Fig. 6(b). Similarly, the left image shows the result of setting the gain of $50 \mathrm{~dB}$ at depth of $1.5-2 \mathrm{~cm}$. Only the blood vessels of lower skin of the mouse are shown clearly. This proves that the system can image targeted depth range of living tissue.

\section{Conclusion}

We successfully established a PAI system based on a pulsed laser and clinical USI system with a commercial US transducer. By utilizing a 3D-printing mold with black hair and a centrifugal tube filled with black ink as the optical absorbers, we reconstructed PA images through the DAS algorithm, which sufficiently justifies the feasibility of the system implementation. For PA signal acquisition, we put forward the method of providing variable gain for different depths. The results obtained using the proposed method showed better resolution and contrast than when using $50 \mathrm{~dB}$ gain for all depths. The depth range resolution was $\sim 1 \mathrm{~mm}$, and the maximum imaging depth of our system reached $\sim 4.6 \mathrm{~cm}$. To further testify the function of variable gain for different depths, we imaged the sagittal section of the body of in vivo nude mice. Tiny blood vessels of the mice were revealed and targeted depth range of living tissue can be acquired.

Comparing with other systems, the imaging depth of our PAI system is superb. The imaging depth of the programmable system ${ }^{1}$ of Kim et al. 
and the system with compact laser21 of Wang is, respectively, $\sim 4.6 \mathrm{~cm}$ and $\sim 4.1 \mathrm{~cm}$. However, specific exogenous dyes were used during their experiments while our system reached $\sim 4.6 \mathrm{~cm}$ only by pure black ink as optical absorber. In addition, the clinical linear array transducer of our system can collect multi-channel signals, which increases the efficiency of signal acquisition.

The resolution of $\mathrm{PA}$ images of our system is expected to be improved. Furthermore, the system is not capable of providing tomographic images for samples. In future study, we aim to focus on improving image quality, especially lateral resolution, by optimizing the DAS algorithm and by implementing other algorithms. Besides, by using shifting device for the probe and improving image reconstruction algorithm, the system is expected to implement PA tomography. Combining with inherent advantages, the system can be applied to small absorbers' imaging. Because the absorbance of hemoglobin is relatively high with the excitation of $728 \mathrm{~nm}$ laser, the system is promising to be used in blood imaging in human tissue for clinical diagnoses and imaging.

\section{Conflict of Interest}

The authors have no relevant financial interests in this article and no potential conflicts of interest to disclose.

\section{Acknowledgments}

This research was made possible with the financial support from National Natural Science Foundation of China (NSFC) (61675113, 61527808, 81401539, and 31271056), Science and Technology Research Program of Shenzhen City (JSGG20150331151536448, JCYJ20160428182247170, and JCYJ2017041217025 5060 ), Shenzhen basic research layout project (JCYJ2 0160324163759208) and the projects in Shenzhen Medical Engineering Laboratory For Human Auditory-equilibrium Function. Besides, we would like to thank Editage [www.editage.cn] for English language editing.

\section{References}

1. J. Kim, S. Park, Y. Jung, S. Chang, J. Park, Y. M. Zhang, J. F. Lovell, C. Kim, "Programmable real-time clinical photoacoustic and ultrasound imaging system," Sci. Rep. 6(11), 35137 (2016).

2. K. Sivasubramanian, M. Pramanik, "High frame rate photoacoustic imaging at 7000 frames per second using clinical ultrasound system," Biomed. Opt. Express 7(2), 312-323 (2016).

3. A. G. Bell, "Selenium and the photophone," Nature 22, 500-503 (1880).

4. L. V. Wang, "Multiscale photoacoustic microscopy and computed tomography," Nat. Photonics 3(9), 503-509 (2009).

5. L. H. V. Wang, S. Hu, "Photoacoustic tomography: In vivo imaging from organelles to organs," Science 335(6075), 1458-1462 (2012).

6. S. Zackrisson, S. van de Ven, S. S. Gambhir, "Light in and sound out: Emerging translational strategies for photoacoustic imaging," Cancer Res. 74(4), 979-1004 (2014).

7. X. D. Wang, Y. J. Pang, G. Ku, X. Y. Xie, G. Stoica, L. H. V. Wang, "Noninvasive laser-induced photoacoustic tomography for structural and functional in vivo imaging of the brain," Nat. Biotechnol. 21(7), 803-806 (2003).

8. J. Gamelin, A. Maurudis, A. Aguirre, F. Huang, P. Y. Guo, L. V. Wang, Q. Zhu, "A real-time photoacoustic tomography system for small animals," Opt. Express 17(13), 10489-10498 (2009).

9. M. Pramanik, G. Ku, C. H. Li, L. H. V. Wang, "Design and evaluation of a novel breast cancer detection system combining both thermoacoustic, (TA) and photoacoustic (PA) tomography," Med. Phys. 35(6), 2218-2223 (2008).

10. A. A. Oraevsky, E. V. Savateeva, S. V. Solomatin, A. A. Karabutov, V. G. Andreev, Z. Gatalica, T. Khamapirad, P. M. Henrichs, "Optoacoustic imaging of blood for visualization and diagnostics of breast cancer," Proc. SPIE 4618, 81-94 (2002).

11. K. H. Song, E. W. Stein, J. A. Margenthaler, L. V. Wang, "Noninvasive photoacoustic identification of sentinel lymph nodes containing methylene blue in vivo in a rat model," J. Biomed. Opt. 13(5), 054033 (2008).

12. K. H. Song, C. Kim, K. Maslov, L. V. Wang, "Noninvasive in vivo spectroscopic nanorod-contrast photoacoustic mapping of sentinel lymph nodes," Eur. J. Radiol. 70(2), 227-231 (2009).

13. C. Passmann, H. Ermert, "A $100-\mathrm{MHz}$ ultrasound imaging system for dermatologic and ophthalmologic diagnostics," IEEE Trans. Ultrason. Ferroelectr. Freq. Control 43(4), 545-552 (1996).

14. T. Misaridis, J. A. Jensen, "Use of modulated excitation signals in medical ultrasound. Part I: Basic concepts and expected benefits," IEEE Trans. Ultrason. Ferroelectr. Freq. Control 52(2), 177-191 (2005). 
15. S. J. Jung, S. K. Hong, O. K. Kwon, "Low-power lownoise amplifier using attenuation-adaptive noise control for ultrasound imaging systems," IEEE Trans. Biomed. Circuits Syst. 11(1), 108-116 (2017).

16. AFE5809 fully integrated, Texas Instruments Incorporated, Dallas, Texas, USA, http://www.ti.com/ product AFE5809/datasheet (2014), pp. 33-35.

17. G. Marquez, L. H. V. Wang, S. P. Lin, J. A. Schwartz, S. L. Thomsen, "Anisotropy in the absorption and scattering spectra of chicken breast tissue," Appl. Optics 37(4), 798-804 (1998).

18. J. L. Sandell, T. C. Zhu, "A review of in vivo optical properties of human tissues and its impact on PDT," J. Biophotonics 4(11-12), 773-787 (2011).

19. American Natianal Standard for Safe Use of Lasers (Laser Institute of America, 2007), pp. 70-77.
20. C. Kim, T. N. Erpelding, L. Jankovic, L. V. Wang, "Performance benchmarks of an array-based handheld photoacoustic probe adapted from a clinical ultrasound system for non-invasive sentinel lymph node imaging", Philos. Trans. R. Soc. A, Math. Phys. Eng. Sci. 369(1955), 4644-4650 (2011).

21. D. P. Wang, Y. H. Wang, W. R. Wang, D. D. Luo, U. Chitgupi, J. M. Geng, Y. Zhou, L. D. Wang, J. F. Lovell, J. Xia, "Deep tissue photoacoustic computed tomography with a fast and compact laser system," Biomed. Opt. Express 8(1), 112-123 (2017).

22. G. Ku, L. H. V. Wang, "Deeply penetrating photoacoustic tomography in biological tissues enhanced with an optical contrast agent," Opt. Lett. 30(5), 507-509 (2005). 